\title{
Correction of Mesially Impacted Lower Second Molar
}

\author{
Ahmet Arif Celebi ${ }^{\mathrm{a}, \mathrm{b}}$, Ibrahim Erhan Gelgor ${ }^{\mathrm{a}}$, Bulent Catalbas ${ }^{\mathrm{a}}$
}

\begin{abstract}
Impaction of the lower second molar is not a common problem, but it is very challenging for both orthodontist and oral surgeon. We report the case of patient who complained of a delay in eruption of both the upper and lower second molars. A panoramic radiograph revealed the presence of all permanent teeth and a severe mesial inclination of the left lower second molars and developing third molars. A removable appliance with an uprighting spring was fixed mouth. After two months, a miniscrew was applied maxillar posterior buccal area. And then, an elastic chain was put between miniscrew and lower second molar. Within a period of 4 months the tooth was completely upright. After 5 months, a panoramic radiograph revealed complete uprighting of the tooth, with bone regeneration. Orthodontic uprighting techniques using miniscrews are more advantageous and offer better long term prognosis than surgical uprighting. The timing of treatment and the biomechanics involved determine the success.
\end{abstract}

Keywords: Mandibular second molar; Miniscrew; Impaction; Uprighting spring

\section{Introduction}

The incidence impaction of the permanent lower second molar lies between $0.6 / 1000$ [1] and 3/1000 [2]. Impaction of the lower second molar is a rare condition, but when it occurs it

Manuscript accepted for publication August 10, 2011

${ }^{a}$ Kirikkale University, Faculty of Dentistry, Department of Orthodontics, Kirikkale, Turkey

${ }^{\mathrm{b}}$ Corresponding author: Ahmet Arif CELEBI, Kirikkale University, Faculty of Dentistry, Department of Orthodontics, Dishekimligi, 692. Sok. No. 31, 71200 Merkez, Kirikkale, Turkey.

Email: arcedent@yahoo.com

doi:10.4021/jmc272w may present a clinical problem for the orthodontist and oral surgeon. Unilateral impaction of the mandibular second molar is more common than bilateral impaction. They are found more often in men than in women and more frequently on the right side than on the left as a mesially inclined [3].

During the last decade, the introduction of miniscrews for immediate loading has changed the clinical and biomechanical approach to the orthodontic problems. Gelgor et al [4], Lee et al [5], Park et al [6], and Giancotti et al [7] have shown the use of titanium miniscrews for immediate loading as an alternative anchorage system and discussed possible sites of insertion. Park et al reported molar uprighting by means of microimplant anchorage in patients with mesially tipped second molars due to the loss of the adjacent first molar [8]. Using titanium miniscrews as absolute anchorage offers several advantages and can aid in achieving an optimal treatment outcome [7]. Freudenthaler et al inserted 12 titanium bicortical screws horizontally as anchorage for mandibular molar protraction in 8 patients [8]. The low frequency of mesially impacted second molars has kept the clinical significance of this anomaly quite low [9]. Therefore, little information is available in the dental literature. Surgical treatment is often recommended [10].

But, orthodontic uprighting may be a better alternative, with a lower risk to the tooth. The aim of this article is to describe our experience that is using of removable appliance with uprighting spring and miniscrew, respectively.

\section{Case Report}

A 15-year-old boy was referred for orthodontic consultation and treatment. Clinical examination revealed Class I interarch relationship (Fig. 1a, b). Examination of the orthopantomograph revealed the presence of all permanent teeth, including developing third molars. The mandibular left permanent second molar were tipped mesially and obliquely impacted under the distal bulge of the permanent first molars. The developing mandibular third molar buds were on top of distal roots of the second molars (Fig. 2).

The treatment plan was gaining of the tooth to dental arch. Under local anesthesia, the impacted mandibular left 

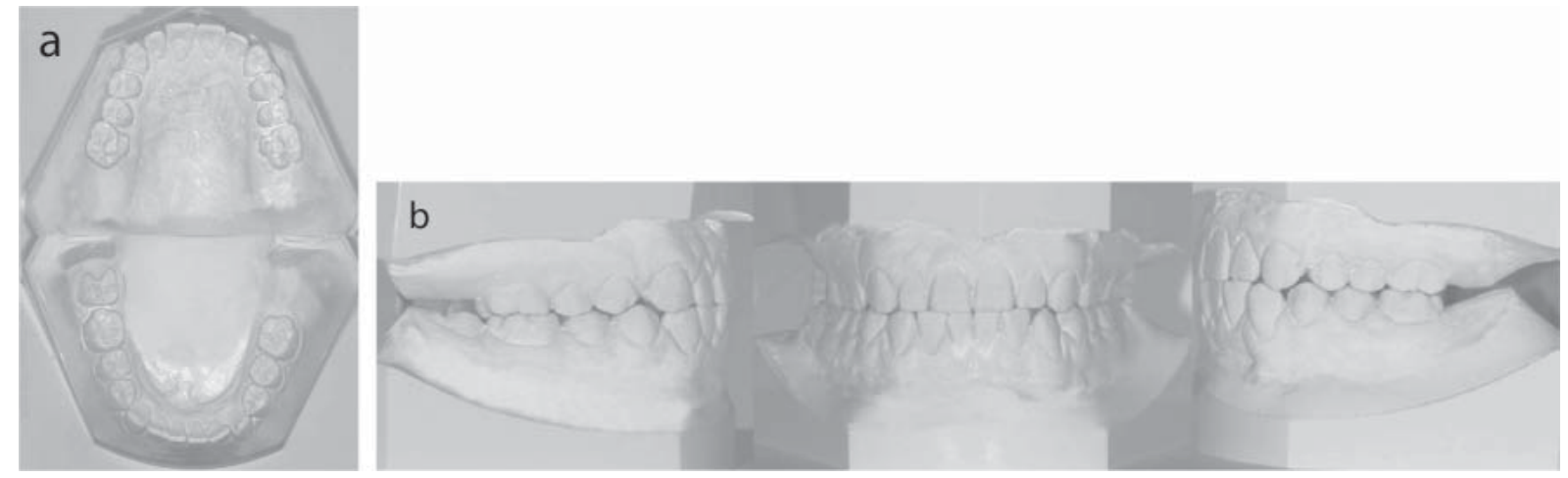

Figure 1. Pre-treatment cast photographs of a 15-year-old male patient ( $a, b)$.

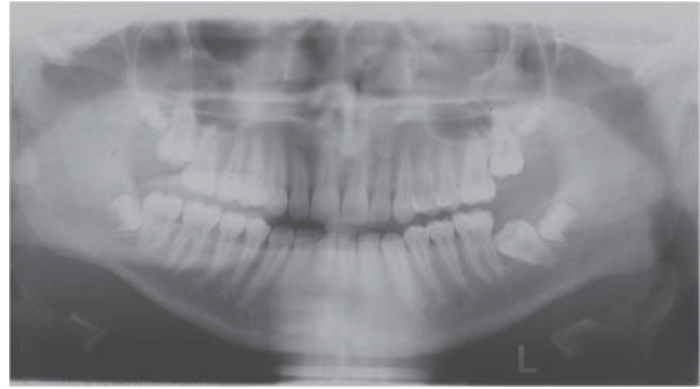

Figure 2. Pre-treatment panoramic radiograph of the patient.

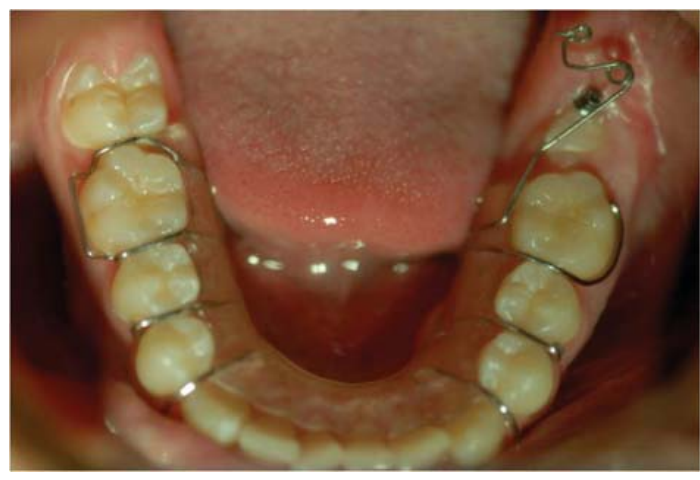

Figure 3. The removable appliance with an uprighting spring.

second molar was surgically exposed and the mucoperiosteum reflected just enough to expose the distobuccal cusp of the tooth. At the same time, the tooth was carefully etched, with due consideration for prevention of soft tissue injury. In spite of the difficulty in controlling moisture, a button was bonded and a ligature wire bended top of the button. Two weeks later, a removable appliance with an uprighting spring that aim of distal uprighting of tooth, was fixed mouth and used two months. The uprighting spring was made up of 0.8 mm stainless wire (Fig. 3).

After using of uprighting spring, a mini screw made of pure medical titanium was used for buccal and vertical

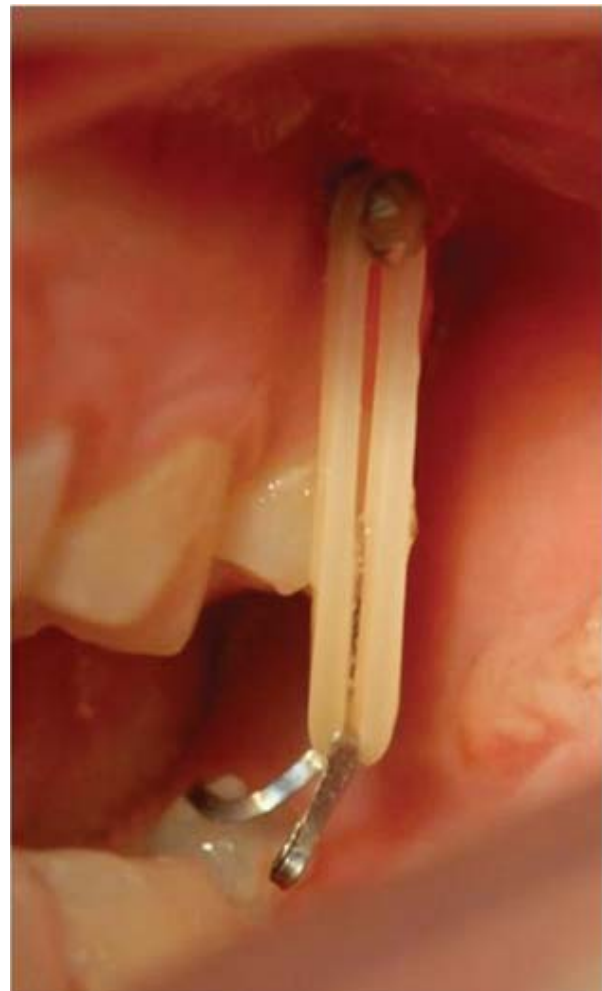

Figure 4. The buccal mini screw for extrusion of lower left second molar.

uprighting. The miniscrew had $1.8 \mathrm{~mm}$ diameter and $8 \mathrm{~mm}$ lengths (Dewimed, Tuttlingen). The screw was inserted under local anesthesia and implanted into buccal side of between the first and second maxillary left molar's roots. When occlusal surface of second molar appear, a hook made 0.016 $\times 0.022$ " stainless wire was adhered. After initial healing of the soft tissue around the mini screw (after 1 week) an elastic chain (3/16, 4 oz.) was applied between the buccal mini screw and the wire on the occlusal surface of mandibular second molar (Fig. 4).

The patient was scheduled for follow-ups every 2 weeks to control anchorage and the movements of the impacted 


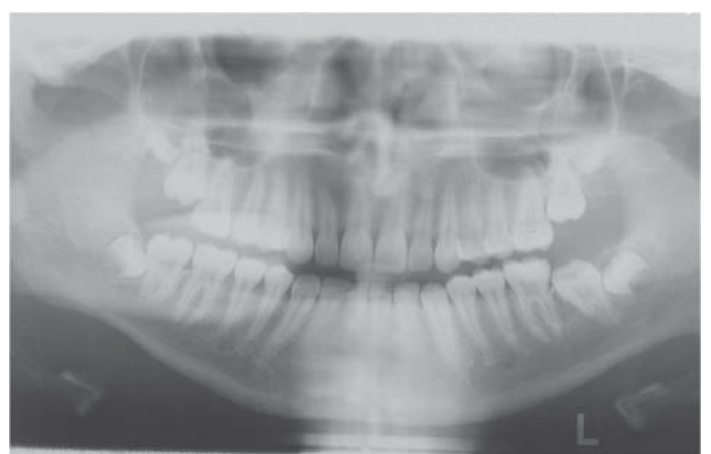

Figure 5. The panoramic radiograph 5 months after the beginning of treatment.

teeth. The hook was changed according to movement of the tooth. After two months, elastic chain altered (3/16, 6 oz.).

5 months after the beginning of treatment, a satisfactory lower second molar inclination was achieved (Fig. 5). At the eighth month, a corrected inclination of the impacted molars was obtained, and was confirmed on the post-treatment panoramic radiograph (Fig. 6) and intraoral photograph (Fig. 7). It was understood that there was no need any orthodontic treatment for this patient.

\section{Discussion}

The proper time to treat the impactions is when the patient is 11 - 14 years old, during early adolescence, when the second molar root formation is still incomplete and before complete development of mandibular third molars [9]. In the present case, the patient was 15 years old, the second molar root formation was incomplete and the third molar was not completely developed.

Mesial inclination of the impacted mandibular second molar is much more common than distal inclination. In fact, most of the cases reported in the literature show the unerupted mandibular second molar mesially inclined in a oblique or

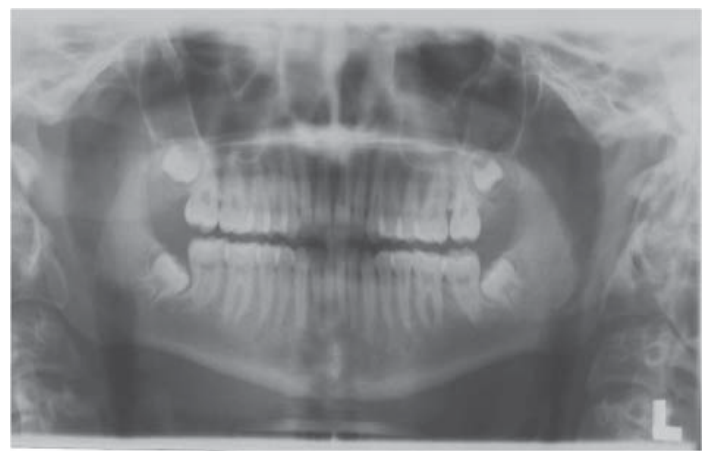

Figure 6. The post-treatment panoramic radiograph of the patient.

horizontal position [3]. It was reported inverted in only one case, with the crown directed toward the border of the mandible [11]. There are many different treatment options in the literature [9, 12]. The significant advantage of orthodontic uprighting technique is the distal tipping and uprighting of the impacted tooth without the necessity of surgical assistance, bone removal or splinting. In the case presented two systems for uprighting were combined. One of them is removable appliance with uprighting springs for distal movement of the molars, another is miniscrew for buccal and vertical movement of it. By using this method, impacted molars will upright within 4 - 8 months.

Advantages of the method: 1), Because of the use of light and intermittent force will minimize the side effect on the anchor molars; 2), Although surgical uprighting of impacted mandibular second molar appear to be a quick and easy procedure, orthodontic uprighting techniques are more advantageous and offer a better long term prognosis with no adverse pulpal or periodontal risk to the tooth or supporting structures; 3), Using of removable appliance with uprighting spring provides distal movement of the mandibular second molar, especially. However, the elastic chain is fixed between the wire on the occlusal surface of mandibular second molar and miniscrew in upper jaw posterior area, transverse

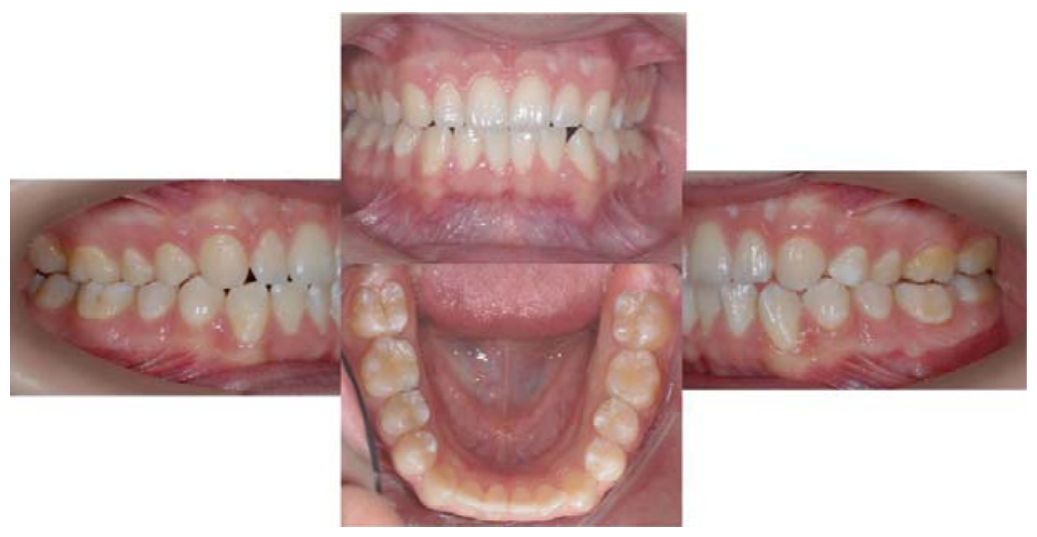

Figure 7. Post-treatment intraoral photograph of the patient. 
plane result in buccal tipping of impacted molar crown and lingual tipping of root of impacted molar and vertical plane result extrusion of the tooth; 4), The appliance eliminated the need for any application other teeth.

Disadvantages of the method: 1) This method is a complex to upright full impacted second molars; 2) A hygienic mouth was not provided by patient; 3) This method can be completely inefficient if the patient cooperation with removable appliance and intermaxillary elastics is poor.

In conclusion, with early diagnosis and recognation of the potentially developing impaction, practitioners are alerted to initiate adequate corrective measures. Second molar impaction is a very difficult case that requires proper clinical, radiological and biomechanical management and a good appliance selection for successful treatment results.

\section{Conflict of Interest}

None

\section{References}

1. Grover PS, Lorton L. The incidence of unerupted permanent teeth and related clinical cases. Oral Surg Oral Med Oral Pathol. 1985;59(4):420-425.

2. Johnsen DC. Prevalence of delayed emergence of permanent teeth as a result of local factors. J Am Dent Assoc. 1977;94(1):100-106.

3. Wellfelt B, Varpio M. Disturbed eruption of the perma- nent lower second molar: treatment and results. ASDC J Dent Child. 1988;55(3):183-189.

4. Gelgor IE, Buyukyilmaz T, Karaman AI, Dolanmaz D, Kalayci A. Intraosseous screw-supported upper molar distalization. Angle Orthod. 2004;74(6):838-850.

5. Lee JS, Park HS, Kyung HM. Micro-implant anchorage for lingual treatment of a skeletal Class II malocclusion. J Clin Orthod. 2001;35(10):643-647; quiz 620.

6. Park HS, Bae SM, Kyung HM, Sung JH. Micro-implant anchorage for treatment of skeletal Class I bialveolar protrusion. J Clin Orthod. 2001;35(7):417-422.

7. Giancotti A, Arcuri C, Barlattani A. Treatment of ectopic mandibular second molar with titanium miniscrews. Am J Orthod Dentofacial Orthop. 2004;126(1):113-117.

8. Freudenthaler JW, Haas R, Bantleon HP. Bicortical titanium screws for critical orthodontic anchorage in the mandible: a preliminary report on clinical applications. Clin Oral Implants Res. 2001;12(4):358-363.

9. Shapira Y, Borell G, Nahlieli O, Kuftinec MM. Uprighting mesially impacted mandibular permanent second molars. Angle Orthod. 1998;68(2):173-178.

10. Davis WH, Patakas BM, Kaminishi RM, Parsch NE. Surgically uprighting and grafting mandibular second molars. Am J Orthod. 1976;69(5):555-561.

11. Fernandes HA. Unerupted maxillary anteriors. Unerupted, inverted mandibular second molar. Case reports. J All India Dent Assoc. 1965;37(8):269.

12. McAboy CP, Grumet JT, Siegel EB, Iacopino AM. Surgical uprighting and repositioning of severely impacted mandibular second molars. J Am Dent Assoc. 2003;134(11):1459-1462. 